\title{
Enfoques en el currículo, la formación docente y metodología en la enseñanza y aprendizaje del inglés: una revisión de la bibliografía y análisis de resultados
}

Arán Sánchez, Ana; Arzola Franco, David Manuel; Ríos Cepeda, Vera Lucía

Enfoques en el currículo, la formación docente y metodología en la enseñanza y aprendizaje del inglés: una revisión de la bibliografía y análisis de resultados

Revista Educación, vol. 46, núm. 1, 2022

Universidad de Costa Rica, Costa Rica

Disponible en: https://www.redalyc.org/articulo.oa?id=44068165024

DOI: https://doi.org/10.15517/revedu.v46i1.45048

\section{(c) (1) $\Theta$}

Esta obra está bajo una Licencia Creative Commons Atribución-NoComercial-SinDerivar 3.0 Internacional. 
Revisiones Bibliográficas

\title{
Enfoques en el currículo, la formación docente y metodología en la enseñanza y aprendizaje del inglés: una revisión de la bibliografía y análisis de resultados
}

\author{
Approaches to English Education Curriculums, Teacher Training and Methodology: A Literary Review and Results \\ Analysis \\ Ana Arán Sánchez \\ Escuela Normal Rural Ricardo Flores Magón, México \\ ana.aran@enrrfm.edu.mx \\ DOI: https://doi.org/10.15517/revedu.v46i1.45048 \\ Redalyc: https://www.redalyc.org/articulo.oa? \\ $\mathrm{id}=44068165024$
}

iD https://orcid.org/0000-0001-7149-3461

David Manuel Arzola Franco

Centro de Investigación y Docencia, México

david.arzola@cid.edu.mx

iD https://orcid.org/0000-0003-2285-099X

Vera Lucia Rios Cepeda

Escuela Normal Rural Ricardo Flores Magón, México

vera.rios@enrrfm.edu.mx

(iD) https://orcid.org/0000-0002-3919-838X

Recepción: 25 Enero 2021

Aprobación: 25 Mayo 2021

\section{RESUMEN:}

El inglés es el idioma que más se enseña a nivel mundial y, debido a su relevancia en diferentes ámbitos como el económico, académico, laboral y de investigación; se le considera la lengua franca. Dada su importancia, es pertinente analizar diferentes cuestiones que forman parte de su proceso de enseñanza y aprendizaje, tales como el currículo, la metodología de enseñanza y la formación docente a partir de los enfoques que guían estos ámbitos. El documento que a continuación se presenta, tiene como propósito revisar las contribuciones al campo de conocimiento de esfuerzos innovadores y actuales a nivel nacional e internacional, comprendida entre los años 2005 y 2020. Su objetivo es generar un corpus de información que permita construir una visión de conjunto sobre este fenómeno, con el uso de la metodología de investigación documental. La revisión de la bibliografía evidencia un uso predominante de metodologías de enseñanza de tipo activa, en las que se destacan, a su vez, el empleo de las tecnologías de la comunicación (TIC) en el aula. Se enfatiza el uso predominante del enfoque comunicativo; sin embargo, se exponen otras propuestas de alternativas pedagógicas situadas. También se revela la falta de articulación entre el currículo formal y el currículo en acción, en aspectos como la evaluación, y el rol que el currículo oculto juega para transmitir ideas. Finalmente, se reflexiona sobre el desarrollo de programas de formación docente que contengan elementos de vanguardia para estar a la par de los cambios sociales. Se sugiere desarrollar capacitaciones de formación continua para que el profesorado conozca los planes de estudio y los puedan llevar a cabo en sus aulas de manera congruente a través de un análisis crítico de este, al igual que la capacitación en metodologías activas y que incluyan el uso de las tecnologías de la información.

Palabras ClaVE: Enfoques, Formación docente, Metodología, Enseñanza y aprendizaje, Inglés.

\section{Abstract:}

English is the language that is taught the most worldwide and considered to be a lingua franca due to its relevance in fields such as economy, academia, labor and research. Given its prevalence, it makes sense to analyze aspects associated with the teaching and learning process, including the curriculum, teaching methodology and teacher training. The objective of this document is to review innovative national and international contributions made to the field of English education from 2005 to 2020 with the goal of building an overview based on documentary research methods. A literature review reveals the predominant use of active teaching methods, highlighting the use of information and communication technologies (ICT) in the classroom and the predominant use of a communicative approach, based on a diverging gamut of pedagogical proposals. Yet, there is an overall disparity between formal and action curriculums, regarding assessment procedures and the role that hidden curriculums plays in transmitting ideas, 
emphasizing the need to give more thought to the development of teacher training programs and include vanguard elements aligned and updated to social trends. Ongoing teacher training programs must be developed which allow teachers to coherently use new syllabuses in the classrooms based on previous critical analysis and better prepare in the use of active methodologies that incorporate information and communication technologies.

KEYWORDS: Approaches, Teacher Training, Methodology, Teaching and Learning, English.

\section{INTRODUCCIÓN}

El inglés es la lengua que más se enseña en los diferentes niveles educativos a nivel mundial (ChávezZambrano et al., 2017), lo que genera amplia gama de estrategias, necesidades y formas de abordarlo en los diferentes contextos (académicos, geográficos, sociales, económicos o culturales). La innegable realidad es que, desde el colonialismo británico y el desarrollo de Estados Unidos como potencia económica y militar después de la segunda guerra mundial (Gutiérrez y Landeros, 2010), junto con la actual globalización tecnológica y económica, el inglés es una de las lenguas más utilizadas a nivel internacional, y suele definirse como el idioma global o la lengua franca (Quezada-Narvaéz, 2011; Jenkins et al., 2011).

Con todo lo anterior, se considera que aprender una lengua de tan amplio uso provee beneficios al estudiante, desde el acceso a un mayor abanico de opciones profesionales, una comprensión más amplia, tanto de su propia cultura como de las ajenas, hasta el desarrollo de la creatividad y la resolución de problemas complejos (Marcos, 1997). Además, permite a la persona aprendiz acceder a información sobre diferentes eventos internacionales, medios de comunicación y bibliografía académica, que se suelen presentar en inglés (Chávez-Zambrano et al., 2017).

En consideración de la amplitud y complejidad de esta temática, surgen inquietudes relacionadas con la manera en que se está abordando la enseñanza y el aprendizaje del inglés desde la investigación educativa, explorar las experiencias de otras personas y aprender de ellas. Por ejemplo, conocer los métodos de enseñanza y los enfoques a los que se adscriben; así como la manera en que se traducen en didácticas particulares, procesos de evaluación y los roles que desempeñan docentes y estudiantes. También está el interés por analizar los componentes del currículum de la enseñanza de la lengua extranjera (competencias, aprendizajes esperados, contenidos y materiales), así como sus enfoques de acuerdo a las teorías de adquisición del idioma en los cuales se basan y los procesos de formación inicial y docente del profesorado de lengua extranjera.

Se revisaron artículos de investigación en torno a esas temáticas, al igual que documentos cuyo propósito es tratar la cuestión del estado del arte en la enseñanza y aprendizaje del idioma inglés. Se encontraron estudios que abarcan de manera general este fenómeno, ya sea a nivel global (Garton et al., 2011) o por zonas geográficas específicas, como algunas regiones africanas (Olufunmilyo, 2014) y países europeos de acuerdo a periodos históricos particulares (Smith y McLelland, 2018).

La mirada también puede ser crítica, al revisar a profundidad las opciones de formación docente y cuestionar, posteriormente, la deficiencia en la preparación para el profesorado futuro de lengua extranjera (Farell, 2015). A su vez, se han realizado esfuerzos para analizar y presentar el estado del arte de aspectos específicos relacionados con la lengua, como los métodos de enseñanza (Salmani-Nodoushan, 2006), la instrucción enfocada a la pronunciación (Thomson y Derwin, 2014) o el desarrollo de la competencia escrita (Hinkel, 2011).

Por lo tanto, el presente trabajo aborda de manera particular las publicaciones recientes que abarcan las temáticas de enfoques curriculares, metodológicos y de formación docente dentro del proceso de enseñanza y aprendizaje del inglés. Su propósito es revisar las contribuciones al campo de conocimiento de cada investigación, con el objetivo de generar un corpus de información que permita construir una visión de conjunto sobre este fenómeno. 


\section{MARCo TEÓRICo}

El análisis de la literatura relacionada con la enseñanza y el aprendizaje de la lengua inglesa, se enfoca en la necesidad de construir una mirada crítica en torno a un fenómeno que se ha caracterizado por desarrollarse a la luz de tendencias centradas en la uniformidad metodológica, heredera de las aspiraciones universalistas del pensamiento científico que se gestó a finales del siglo XIX.

En el siglo XX, se pueden identificar varios periodos dominados por el enfoque estructuralista, donde la gramática y el vocabulario ocupan un lugar central y las necesidades del alumnado "son identificadas únicamente en término de necesidades lingüísticas" (Branda, 2017, p.101). Sin embargo, a partir de los años setenta, inspirado en la sociolingüística, es el enfoque comunicativo el que se torna referente obligado para las definiciones curriculares (Richards, 2001); esto lo ubica en una posición hegemónica con respecto a otras perspectivas emergentes. Aunque parten de principios dispares, tanto el estructuralismo como el enfoque comunicativo se caracterizan por su ahistoricidad, que desdeña la relevancia de los elementos contextuales, situados culturalmente, mientras se exaltan los principios de neutralidad y objetividad científica.

No obstante, en el contexto contemporáneo, caracterizado por los movimientos posmodernos y deconstruccionistas, han surgido propuestas contrahegemónicas que buscan, dentro de sus propias tradiciones educativas, la inspiración para proponer alternativas pedagógicas situadas (Ouyang, 2000), que respondan a las necesidades y expectativas del estudiantado de acuerdo con el contexto en el que se desenvuelven, sobre todo si se considera "que un idioma extranjero no es un vehículo de comunicación neutro sino que comporta ideologías y valores que tienen un impacto social y político" (Muñoz, 2010, p. 83). Esta carga axiológica, ideológica y política, latente en las propuestas curriculares, es lo que se define como curriculum oculto (Aranda et al., 2016).

En este sentido, la pedagogía crítica, particularmente aquella que emana del escenario latinoamericano (Vargas, 2017), se convierte en un referente conceptual novedoso para el presente trabajo, en tanto propone el desarrollo de formas emic para enfrentar los problemas educativos relacionados con la enseñanza de lenguas extranjeras, con el potencial de contribuir en el debate, la reflexión, el empoderamiento de las partes involucradas y la transformación de las prácticas. De tal manera que el alumnado disponga de experiencias lingüísticas enriquecedoras sin que sus identidades sean vulneradas (Noroozisiam y Soozandehfar, 2011).

\section{Estrategia METODológica}

Este trabajo se realizó a través del método de investigación documental, el cual, a pesar de haber estado presente desde los inicios de la investigación social, en ocasiones se le excluye y se utiliza como última opción con respecto a otros métodos (Ahmed, 2010). No obstante, “al representar sistemática y sintéticamente los documentos originales, facilita su recuperación y consulta” (Dulzaides y Molina, 2004, p.3). Este método consiste en "buscar, seleccionar, organizar y analizar un conjunto de materiales escritos para responder una o varias preguntas sobre un tema" (Tobón et al., 2015, p. 9) a través de un proceso de tipo sistemático (Bowen, 2009), metódico y analítico (Pinto, 1989). Asimismo, busca "describir y representar los documentos de forma unificada y sistemática para facilitar su recuperación (Dulzaides y Molina, 2004, p. 2).

El trabajo se desarrolló en dos etapas. La primera, considerada de tipo heurística, consistió en recolectar, seleccionar y clasificar las publicaciones relacionadas con el objeto de estudio. Como estrategia de búsqueda se utilizaron palabras clave para localizar estudios que exploraran cada una de las categorías y que estuvieran dentro del rango de publicaciones consideradas como estudios de frontera, por ser recientes e innovadoras en su tratamiento de la temática de interés.

Para sistematizar y agrupar la información relevante de cada artículo consultado, se utilizó un cuadro de doble entrada que contenía los elementos que propone Medina (2011): datos bibliográficos, problema de investigación, conceptos, sujetos, técnicas e instrumentos, resultados y conclusiones. 
Una segunda etapa se realizó a través de un análisis hermenéutico, a partir de la elaboración de reportes de investigación de cada artículo que previamente se habían leído y analizado, incluyendo citas textuales y comentarios personales acerca del estudio. Se trató de hacer una nueva revisión de la información recopilada para interpretarla desde otra perspectiva, con el objetivo de "mirar los resultados del estudio como vacíos, limitaciones, dificultades, tendencias y logros obtenidos con el fin de presentar el estado actual de la investigación de manera global que permitiera orientar nuevas líneas de investigación" (Guevara, 2016, p. 176). Esto permitió regresar al problema y examinarlo a la luz de lo hallado en la bibliografía revisada, con el objetivo de afinarlo (Medina, 2011).

Para lograr el propósito de este documento, se revisaron 75 investigaciones, comprendidas entre los años 2005 y 2020, llevadas a cabo, principalmente, en Latinoamérica, Norteamérica y Europa, con algunas situadas en países africanos y asiáticos. Siete de estas publicaciones están escritas en inglés y el resto en español. Estos documentos abordan el fenómeno desde diversos enfoques, con una amplia variedad de diseños metodológicos, técnicas e instrumentos. El hecho de revisar indagaciones de diferentes partes del mundo, permite identificar las diversas formas en las que se abordan cuestiones relacionadas con la enseñanza y aprendizaje del idioma de acuerdo al contexto. Por ejemplo, el documento publicado en Sudáfrica explora el análisis del currículo de esta lengua en un país que en su momento fue colonia de Reino Unido, cuyo idioma oficial es el inglés a pesar de tener también cuatro lenguas adicionales; lo cual tiene implicaciones diferentes a las investigaciones que muestran el panorama de Latinoamérica, donde este idioma se considera una lengua extranjera. Esta información se resume en la Tabla 1, la cual se presenta a continuación.

TABLA 1

Investigaciones de acuerdo a país e idioma

\begin{tabular}{lllc}
\hline Región & País & Núm. & Idioma \\
& Colombia & 12 & Español \\
& Ecuador & 12 & \\
& España & 10 & \\
Costa Rica & 7 & \\
& Argentina & 7 & \\
& México & 7 & \\
& Cuba & 5 & \\
& Chile & 4 & \\
& Perú & 3 & \\
\multirow{4}{*}{ Países Anglosajones } & Venezuela & 1 & \\
& Inglaterra & 2 & Inglés \\
Otros & Canadá & 1 & \\
& Estados Unidos & 1 & \\
total & Irán & 2 & \\
\hline
\end{tabular}

Fuente: elaboración propia.

En la Tabla 2, se muestran las investigaciones consultadas en relación con los diversos buscadores académicos empleados para la localización de dichos documentos.

TABLA 2

Investigaciones de acuerdo a los buscadores utilizados

\begin{tabular}{|c|c|c|c|c|c|c|}
\hline 20 & 19 & & 10 & & & 3 \\
\hline
\end{tabular}

Fuente: elaboración propia. 
De las 92 fuentes consultadas para elaborar este documento, se utilizaron 75 para desarrollar las tres categorías de análisis. Si bien, más de la mitad de las investigaciones (57\%) tratan la temática de la metodología, frente al $15 \%$ que versan sobre el currículo y el $18 \%$ sobre la formación docente; cabe resaltar que varias fuentes se utilizaron para enriquecer más de una categoría.

TABLA 3

Investigaciones de acuerdo a las categorías de análisis

\begin{tabular}{llll}
\hline Metodología & $\begin{array}{l}\text { Formación } \\
\text { docente }\end{array}$ & Currículo & Total \\
47 & 15 & 13 & 75 \\
\hline
\end{tabular}

Fuente: elaboración propia.

En la Tabla 3 se muestra la distribución de los artículos de acuerdo a la temática principal que abordan. El artículo abarca las temáticas expuestas, se comienza con una revisión de los enfoques curriculares, para posteriormente revisar los enfoques metodológicos e identificar la manera en que estos dos ámbitos se plasman en los programas de formación del profesorado de Inglés.

\section{Discusión DE RESULTADOS}

\section{El currículo y sus enfoques}

El término currículum hace alusión a una amplia gama de concepciones que han evolucionado a través del tiempo. Para Aranda et al. (2016), se refiere a un "recorrido de formación”, el cual precisa "el porqué, el qué, el cuándo, el cómo, el con quién, y el dónde aprender” (p. 1). Blázquez y Tagle (2010), por su parte, lo definen como "el instrumento fundamental con el que se dota al alumnado y a la sociedad en general por parte de los educadores “(p. 1), mientras que Núñez (2008) lo refiere como la "globalidad del programa" (p. 3). El recorrido conceptual incluye las nociones de currículo prescrito o formal, currículo real o en acción y el currículo oculto.

El currículo prescrito se puede definir como "el programa o documento oficial emanado por el Estado o institución donde están expresados las asignaturas y contenidos que ordenarán los procesos educativos" (Sacristán, 1998, citado por Becerra, 2016, p.186). Por otro lado, sobre el currículo en acción, Agulló (2014), explica que es "la selección concreta de objetivos, contenidos y métodos realizada en el aulacurrículo real-, sea planificada con antelación o no" (p. 3). Finalmente, el currículo oculto se refiere a "el conjunto de contenidos que se transmiten de forma implícita en un contexto educativo" (Aranda et al., 2016, p. 1).

El currículo oculto es capaz de generar actitudes y creencias estereotipadas sobre cuestiones como el género, la raza o las clases sociales (Patarroyo et al., 2004). Como muestra de ello, además del aspecto comunicativo y propiamente académico, se transmite una serie de ideologías y componentes culturales en los libros de texto (Aranda et al., 2016). De igual manera, las directrices del sistema político y económico influyen (Lin, 2008). En este sentido, se sugiere al profesorado estar consciente de este tipo de contenidos que se transmiten a través del currículo oculto, esto por medio de una visión crítica a la hora de implementarlo en el aula.

Los elementos del currículo formal se articulan a través de la planeación didáctica que se implementará en clase; sin embargo, en la bibliografía consultada se constata la distancia entre la planeación y la realidad áulica. Cuando el currículo se convierte en acción, hay discrepancias y una amplia brecha entre los dos procesos, sea al implementarse en educación básica (Mayora y Gutiérrez, 2019; Marmolejo y Mayora, 2020), media superior (Ortega-Auquilla y Minchala-Buri, 2019) o universidad (Becerra, 2016). Frecuentemente, esto sucede por 
una falta de comunicación entre las escuelas formadoras de docentes y las instituciones de educación básica, cuyo contacto, en ocasiones, se reduce a las jornadas de práctica, donde el profesorado futuro se da cuenta de la realidad educativa. Otro aspecto relacionado con esta problemática es el diseño curricular.

Esta falta coherencia entre los planes de estudio y la práctica docente evidencia la compleja trama de relaciones e interacciones que se va tejiendo entre el momento del diseño y el momento de la acción. En el caso de México, muchos son creados por instituciones extranjeras que no conocen la realidad del país (Ramírez et al., 2012).Por esta razón, algunas personas docentes-investigadoras crean propuestas de diseño curricular basadas en las necesidades de aprendizaje e interés en la lengua de su alumnado, así como en atender la demanda laboral (López et al., 2017) o social (Sarmiento et al., 2017); a nivel licenciatura (Rojas, 2018; Cortina, 2013) o posgrado (Janssen et al., 2012). En algunos casos, se opta por no tener un currículo definido, sino diseñar la trayectoria curricular en conjunto (Ruíz et al., 2020).

Para definir el enfoque que guiará el currículo prescrito, las personas diseñadoras se apoyan en las teorías de adquisición de una segunda lengua desarrolladas por la Psicología y la Lingüística. Se encuentran tres enfoques: estructuralista, funcional y de propósitos específicos. La Lingüística Estructural da paso al enfoque estructuralista (EF), el cual se basa en el vocabulario y la gramática; cuando surge la Lingüística Funcional, se centra la atención en la función comunicativa dentro de diferentes contextos (Branda, 2017). Posteriormente, surge Inglés para Propósitos Especificos (IPE), que se fundamenta en el análisis de las necesidades comunicativas del estudiantado con un perfil profesionalizante.

El enfoque comunicativo tiene sus antecedentes en el EF y el IPE. Son varias las personas investigadoras que lo consideran como el óptimo para desarrollar las habilidades de comprensión y expresión (Galvañy y Martín, 2011) y, en la actualidad, es el más aceptado en los diferentes niveles educativos a nivel internacional para trabajar la adquisición de una lengua adicional. Dicho enfoque prioriza el fortalecimiento de la competencia comunicativa (Luna-Hernández et al., 2014; Recino et al., 2014; Cedeño-Álava et al., 2020); es de tipo práctico, y se construye a través del uso (González y Moyetta, 2014), ya que deja la gramática en un lugar menos relevante y potencializa las oportunidades de aplicar el conocimiento (Cruz et al., 2016).

La evolución del enfoque comunicativo desemboca en el enfoque basado en tareas (EBT), el cual busca que el alumnado utilice el idioma en contextos comunicativos a través de diferentes labores que les son asignadas (Tejeda y Borrero, 2019). Cada estudiante elige la forma que le resulte más adecuada para realizar una tarea que implique comunicarse; se fomenta la cooperación, responsabilidad y socialización (Cortina, 2013) en un encuentro práctico con el idioma y contextualizado, lo cual puede favorecer la motivación y actitud positiva hacia este.

Dentro de las perspectivas actuales para la enseñanza de una lengua extranjera, se encuentra el enfoque lúdico. Se puede considerar como una de las pioneras que estableció las bases de esta tendencia a Montessori, cuyo método promueve la independencia en el alumnado y su capacidad para resolver problemas (Ghaffari, et al., 2017).

Pudiera parecer que las orientaciones más tradicionales, como la gramática-traducción o el audio lingual, no resultan relevantes en la actualidad, pero también hay quienes las defienden, como Correa (2019), que plantea la necesidad de revalorizar los enfoques más conservadores de enseñanza. En la misma línea, existe una crítica a los enfoques que excluyen por completo la gramática, ya que esta es inherente al aprendizaje de una segunda lengua (Ming, 2012). A su vez, hay personas expertas que, a pesar de basarse en el enfoque comunicativo para diseñar su metodología de clase, incorporan actividades de práctica y repetición, que se asocian a los enfoques más tradicionales (Lin, 2008).

A continuación, se considera pertinente abordar la manera en la que se enseña el idioma inglés, esto por medio de una revisión de los principales enfoques, las metodologías que se derivan de ellos y las formas de evaluar que plantea cada una de ellas. 


\section{Enfoques y metodologias de enseñanza}

Existen diversos aspectos que forman parte de los procesos de enseñanza y aprendizaje que para el profesorado son más que un cúmulo de conocimientos que hay que transferir a las personas estudiantes. En el caso particular del aprendizaje de una lengua extranjera, no se reduce a los contenidos conceptuales o teóricos, si bien son importantes, su función radica en poderlos enlazar con los procedimentales, al fomentar el desarrollo de competencias comunicativas.

Una manera en la que se pueden potencializar las habilidades básicas que constituyen la competencia comunicativa es a través del uso de la tecnología, que, a su vez, desarrolla las competencias digitales (GarcíaValcárcel, Basilotta y Mulas, 2016; Rodríguez y Gómez, 2017). Adicionalmente, las TIC (Tecnologías de la Información y la Comunicación) y las TAC (Tecnologías del Aprendizaje y el Conocimiento) desempeñan también un papel importante para reducir la brecha lingüística (Gómez-Parra y Huertas-Abril, 2019; EspejoMohedano y Palacios-Hidalgo, 2019). Tal es el caso de la metodología B-Learning o Blended Learning (en español aprendizaje híbrido), la cual incrementa el nivel de competencia de las habilidades de inglés (Roldán, 2018), especialmente la comunicación oral (Sagástegui et al., 2018; Rojas y Montes de Oca, 2018) y, en general, la mejora del proceso de enseñanza y aprendizaje de la lengua extranjera (Zambrano et al., 2017; Zambrano y Mendoza, 2018).

Existen otros enfoques contemporáneos de enseñanza, los cuales parten de la lingüística funcional (a diferencia de los que se enfocan en el vocabulario y la gramática con una estructura tradicional), basados en la función comunicativa en diferentes contextos (Branda, 2013). Un ejemplo de ello son los métodos activos, los cuales implican la práctica y la reflexión docente (Oros et al., 2018). Este enfoque es una opción frente al reto de motivar al alumnado en clase, a través del uso de materiales que puedan resultarles significativos (Naranjo y Naranjo, 2017). Como muestra de la metodología activa, está flipped classroom o aula invertida (Aguayo et al., 2019; Martínez-Salas, 2019). Su nombre viene de invertir el papel tradicional de la persona docente como poseedora y transmisora de conocimiento, al dotarle este rol al alumnado. Sin embargo, es importante notar que, debido a su popularidad actual, muchos centros educativos, especialmente en el ámbito privado, afirman utilizar esta metodología y la realidad es que la mayoría de las personas docentes no tienen un conocimiento adecuado de ella y no la implementan en sus aulas.

Igualmente, hay metodologías activas que no fueron creadas expresamente para el aprendizaje de una lengua extranjera, pero pueden utilizarse para lograr ese fin, como el método de proyectos (Mena et al., 2016), el aprendizaje basado en juegos o gamificación (Zepeda-Hernández et al., 2016; Mogrovejo et al., 2019) y el aprendizaje colaborativo (García-Valcárcel et al., 2016; Ortiz-Navarrete y Díaz-Larenas, 2017). Estas formas de enseñanza, toman como principio el contacto funcional con el idioma a través del material auténtico, es decir, materiales que un hablante nativo utilizaría en su vida cotidiana, como periódicos, revistas, programas de televisión etc., lo que favorece la interacción entre el estudiantado y propicia su involucramiento y participación.

Uno de los procesos esenciales que forman parte de la enseñanza es la evaluación; de acuerdo al tipo de metodología implementada, las profesoras y profesores utilizan diversas técnicas y estrategias para valorar el aprendizaje del alumnado y reflexionar acerca de su práctica docente. El auge del enfoque comunicativo se refleja en los procesos de evaluación, centrada principalmente en valorar la expresión oral (Fierro et al., 2016; Aguilera et al., 2017; Martínez et al., 2018). No obstante, se evalúan también de manera particular otras habilidades propias de la lengua, como la comprensión lectora (Ochoa-Vásquez, Ramírez-Montoya, 2017). En este proceso se utiliza tanto la evaluación formativa como sumativa, así como la heteroevaluación, coevaluación y la autoevaluación (Mora, 2011; Rivera y Garrote, 2017), con técnicas como las rúbricas (Dávila et al., 2020) y el portafolio (Angola y Zambrano, 2016).

No obstante, al igual que al analizar y estudiar las metodologías que utiliza el profesorado para la enseñanza del idioma, se observa una falta de articulación entre el currículo real y el formal; los métodos de evaluación 
que se implementan en las aulas no siempre corresponden al enfoque que guía la metodología utilizada; esto evidencia el uso de técnicas asociadas a otro tipo de didáctica. Lo anterior se observa especialmente en las escuelas que afirman implementar el enfoque comunicativo, pero, por falta de capacitación del personal docente y desconocimiento, no se plasma en los procesos de evaluación.

Antes de implementar los enfoques de enseñanza y el currículo de la lengua extranjera en su práctica docente, la persona profesional de la educación adquiere, durante su formación inicial y permanente, el conocimiento teórico indispensable para su ejercicio profesional. En el siguiente apartado se consideran diversos elementos relacionados con la formación de maestras y maestros de lengua extranjera

\section{La formación docente del profesorado de Inglés}

La práctica del profesorado es un proceso en permanente construcción; fuertemente vinculado con la forma en la que se ha aprendido a ser docente. Sin embargo, la formación inicial resulta insuficiente, ya que las maestras y maestros de Inglés requieren actualizarse en las prácticas pedagógicas (Calderón y Mora, 2012), así como incrementar sus habilidades lingüísticas del dominio del idioma (Córdoba et al., 2015). La necesidad de una formación continua es evidente, sin embargo, los programas de formación permanente para el profesorado en servicio suelen centrarse únicamente en la metodología, dejando a un lado el análisis de los programas de estudio y el proceso de aprendizaje del profesorado (Cárdenas et al., 2010), por lo que resulta insuficiente para lograr una capacitación adecuada para este colectivo docente.

Existen diversos enfoques a la hora de formar al futuro personal docente de Inglés. Desde la Pedagogía Crítica se promueve la formación integral en la educación y, a través de la Pedagogía Humanizante (Echeverri et al., 2014), se desarrolla la alfabetización académica y crítica con análisis de referentes teóricos y metodológicos (Estrada y García, 2018) o se centra la atención en los estudios post-coloniales y conceptos como el poder, la representación y la historicidad (Calvete y Laurentis, 2014).

Actualmente, hay dos tendencias que han tenido mucho eco en la formación de docentes que imparten los cursos de lengua extranjera: el enfoque reflexivo y el narrativo. El primero busca que el alumnado constantemente reflexione acerca de sus creencias sobre la lengua y la literacidad (Mora, 2011) y las contrasten o adapten a la institución educativa en la que están practicando (Branda, 2017), con el objetivo de fortalecer las habilidades que ejercen durante su práctica profesional (Badilla et al., 2014). El segundo utiliza los relatos de vida para explorar aspectos como el currículo de la formación inicial (Sarasa, 2018) y su trayectoria académica en esta (Sarasa, 2017), así como el objetivo de generar voces alternativas a las oficiales predominantes, que valoren la democracia, inclusión y diversidad (Laurentis y Porta, 2014).

Cabe mencionar que todos los enfoques de formación docente de vanguardia replantean ciertos principios y prácticas que no consideran viables con los cambios que se han producido en la sociedad y, de manera particular, en el ámbito educativo. Sin embargo, siguen incorporando principios esenciales para capacitar a docentes en la enseñanza de una lengua adicional, principalmente en lo referido al desarrollo de competencias lingüísticas, teorías de adquisición de una lengua y el conocimiento teórico y práctico de los aspectos que forman parte del idioma (semántica, gramática, sintáctica, pragmática, entre otros).

Los enfoques de los programas de formación inicial proveen lineamientos para llevar a cabo la metodología en el proceso de enseñanza y aprendizaje. En este sentido, la variedad que cada universidad o persona catedrática implementa responde al contexto de la institución formadora, así como el centro de práctica: desde el uso de las tecnologías de la información y la comunicación (TIC) con la elaboración de recursos educativos por parte de futuras personas docentes para emplear en sus prácticas profesionales (Quesada, 2015) o el empleo de páginas web, blogs y wikis para (Arias, 2012), hasta el diseño e implementación de un protocolo para observar a las personas practicantes (Batt y Díaz, 2010). A su vez, existen ejemplos de metodologías alternativas e innovadoras para apoyar el aprendizaje del estudiantado (Paineán et al., 2012), 
tales como la didáctica no parametral, la cual tiene como eje fundamental la autonomía (Del Campo, 2012) o el aprendizaje basado en problemas (ABP) (Paineán et al., 2012).

\section{Reflexiones Finales}

La relevancia del inglés como el idioma principal de este mundo globalizado pone en evidencia la necesidad de analizar las investigaciones de frontera que tratan este fenómeno desde diferentes perspectivas metodológicas y contextos geográficos, lo cual permite conocerlo con un mayor grado de profundidad. Explorar las diferentes cuestiones que integran el proceso de enseñanza y aprendizaje de esta lengua favorece la reflexión de la práctica docente, y la posible mejora de esta a través de la socialización de los hallazgos relacionados con el currículo, la formación del profesorado y los enfoques que guían las metodologías de enseñanza de este idioma.

Si bien se realizó una revisión bibliográfica extensa y diversa en cuanto a los paradigmas, enfoques y métodos que enmarcan cada investigación, hubo numerosos documentos que no se pudieron revisar, mayormente artículos europeos y anglosajones, por tener un acceso restringido. Lo anterior impide generar una visión más holística e internacional, de gran relevancia para un tema de interés global debido al estatus del idioma como lengua franca.

Como líneas de investigación a futuro, se sugiere analizar los cambios que están surgiendo a nivel internacional en los sistemas educativos debido a la emergencia sanitaria, para explorar de qué manera repercuten en el currículo, modelos de enseñanza para el aprendizaje del inglés, y si, eventualmente, esos cambios se reflejarán en la formación docente.

La bibliografía revisada aporta hallazgos significativos y relevantes en la educación actual para adquirir una lengua adicional. Sobre la variedad de metodologías de enseñanza existentes, se evidencia una amplia gama de investigaciones que utilizan metodologías activas (se revisaron siete para este documento), tanto las desarrolladas específicamente para el aprendizaje de un idioma como las existentes en general. Se destaca un rol especial también del uso de las tecnologías de la información, presente en diez de las indagaciones analizadas. A partir de los cambios provocados por la pandemia del Covid-19 en los sistemas educativos, estas formas de enseñanza han adquirido mayor relevancia, es probable que su empleo continúe en aumento. Se detecta que el enfoque comunicativo sigue siendo dominante en los estudios, no obstante, se avizoran propuestas alternativas que van emergiendo poco a poco.

Respecto al análisis de los enfoques curriculares, se observa una falta de articulación entre lo establecido en los planes y programas de estudio y la forma de trabajo en el aula (currículo formal y en acción, respectivamente), por lo que se sugiere la socialización del currículo en acción por parte de los maestros y maestras de Inglés de cada institución, con el fin de propiciar la reflexión de la práctica docente. Finalmente, se destaca la evolución en el desarrollo y planteamiento de enfoques y propuestas metodológicas en la formación de docentes de Inglés, que, si bien surgen y se adaptan a los cambios sociales, esto no implica hacer a un lado a las perspectivas que les dieron origen. El desarrollo de los programas de formación de vanguardia apuesta por introducir aspectos como la constante reflexión del futuro profesorado, así como valorar la diversidad de voces y tomar en cuenta diferentes perspectivas y experiencias para contextualizar su aprendizaje.

\section{RECOMENDACIONES}

Sobre la temática del currículo, se detecta una brecha considerable entre lo que plantean los planes y lo que el profesorado lleva a cabo en las aulas. En ocasiones, esto se debe a que una gran parte de los programas son diseñados por instituciones extranjeras que desconocen el contexto sociocultural de los centros educativos. Frente a esta situación, diferentes instancias optan por crear e implementar diseños 
curriculares que respondan a las necesidades e intereses del alumnado, o la demanda laboral del país en cuestión.

También se advierte cómo el currículo oculto transmite actitudes, creencias e ideologías, por ejemplo, desde los libros de texto; cuestión ante la cual el profesorado debe de estar atento y consciente. Se recomienda que el profesorado cuente con una actitud crítica ante los planes y programas, para no simplemente actuar como técnico y transmitirlos de manera literal en el salón de clases.

Sobre la metodología, se considera importante fortalecer la capacitación del profesorado en las metodologías activas, las cuales favorecen la motivación del alumnado y su participación activa en la clase, así como la reflexión docente. Un ejemplo de ello es la metodología del aula invertida. De la misma manera, continuar con la familiarización con las TIC y TAC, ya que es probable que los modelos de enseñanza basados en ellas, tales como el modelo híbrido, continúen utilizándose más allá de la cuarentena causada por el virus Covid-19.

Otro aspecto relevante que se destaca en este ámbito, a partir de la revisión bibliográfica, es la necesidad de que el profesorado evalúe de acuerdo al modelo de enseñanza que está implementando con el estudiantado. En este sentido, se identifica un área de oportunidad que se relaciona con la formación continua del profesorado, para formarlo en técnicas, estrategias y modelos de evaluación de acuerdo al enfoque de guía su didáctica.

Para el diseño de la formación inicial de los docentes de inglés, se sugiere incorporar aspectos de los enfoques de vanguardia, como el narrativo y la pedagogía crítica, los cuales toman en cuenta las características del alumnado actual y los cambios sociales para favorecer los procesos reflexivos en los futuros docentes y el contemplar voces alternativas a las predominantes. En este sentido, se plantea la exploración de ideas como la "vernaculización del inglés" de Kumaramadivelu (2011, citado por Branda, 2017).

Sobre los programas continuos de formación docente, se plantea la necesidad de no centrarse exclusivamente en el conocimiento y dominio de nuevas metodologías, sino también acompañar a las maestras y maestros a comprender e implementar adecuadamente los nuevos planes de estudio producto de las reformas educativas. Asimismo, considerar la necesidad de continuar el refuerzo de sus habilidades lingüísticas, posterior a su paso por la formación inicial.

\section{REFERENCIAS}

Aguayo, M., Bravo, M., Nocetti, A., Concha, L. y Aburto, R. (2019). Perspectiva estudiantil del modelo pedagógico flipped classroom o aula invertida en el aprendizaje del inglés como lengua extranjera. Revista Educación, 43(1), 1-16. http://www.redalyc.org/articulo.oa?id=44057415007

Aguilera, G., Ferrer, E. A., Fiol, A., Olivero, M. y Salgado, I. (2017). Algunas experiencias y resultados de la evaluación de tarea de aprendizaje en la enseñanza del idioma inglés. Revista Cognosis, Revista de Filosofía, Letras y Ciencias de la Educación, 2(1), 7-26. https://doi.org/10.33936/cognosis.v2i1.277

Agulló, J. (2014). Documentos y procesos curriculares: sobre la evaluación curricular y los currículos formales, ocultos y reales en ELE. Revista electrónica de didáctica del español como lengua extranjera, (26), 197-213. https://dialn et.unirioja.es/servlet/articulo?codigo $=4953365$

Ahmed, J. (2010). Documentary Research Method: New Dimensions. Indus Journal of Management and Social Sciences, 4(1), 1-14. https://ideas.repec.org/a/iih/journl/v4y2010i1p1-14.html

Angola, J. y Zambrano, G. (2016). El portafolio de valoración como estrategia de evaluación en la producción escrita del inglés. Acción Pedagógica, 25(1), 48-59. http://www.saber.ula.ve/handle/123456789/43202

Aranda, M. L., Cortés, G. y Ruiz, C. (2016). El currículo oculto en la enseñanza de lenguas extranjeras o "las buenas intenciones de la educación. Relingüistica Aplicada, (19). https://dialnet.unirioja.es/servlet/articulo?codigo=6 005866 
Arias, L. D. (2012). Impacto de un curso mediado por la Web 2.0 en el desarrollo profesional de un grupo de futuros docentes de inglés. Revista Folios, (36), 51-76. http://www.redalyc.org/articulo.oa?id=345932039004

Badilla, I., Ramírez, A., Rizo, L. y Rojas, K. (2014). Estrategias didácticas para promover la autorreflexión de la praxis en los procesos de formación docente. Revista Electrónica Educare, 18(2), 209-231. https://www.scielo.sa.cr/p $\mathrm{df} / \mathrm{ree} / \mathrm{v} 18 \mathrm{n} 2 / \mathrm{a} 11 \mathrm{v} 18 \mathrm{n} 2 . \mathrm{pdf}$

Batt, E. G. y Díaz, C. (2010). Explorando un modelo lingüístico-pedagógico innovador para la enseñanza y aprendizaje del inglés y la formación inicial docente en Chile. Contextos, estudios de humanidades y ciencias sociales, (24), 29-41. https://dialnet.unirioja.es/servlet/articulo?codigo $=3683911$

Blázquez, F.y Tagle, T. (2010). Formación docente: un estudio de las creencias de alumnos y profesores sobre el proceso de enseñanza y aprendizaje del inglés. Revista Iberoamericana de Educación, 54(4), 64-90. https://doi.org/10.3 $5362 /$ rie 5441659

Becerra, T. (2016). El currículo prescrito y el de acción en una clase de inglés como lengua extranjera. Lengua y Habla, 20, 183-199. http://erevistas.saber.ula.ve/index.php/lenguayhabla/article/view/8103/8044

Bowen, G. (2009). Document Analysis as a Qualitative Research Method. Qualitative Research Journal, 9(2), 27-40. https://bit.ly/3onmRWh

Branda, S. (2013). Las Didácticas Específicas en la Formación del Profesorado: el área de Formación Docente en el Profesorado de Inglés en la UNMdp. Revista de Educación, 4(6), 149-162. https://bit.ly/3nnFqrX

Branda, S. (2017). Los distintos enfoques en la enseñanza del inglés como lengua extranjera y segunda: nuevas tendencias en la literatura contemporánea. Revista de Educación, (11), 99-112. https://bit.ly/2Xkz5mC

Calderón, R. y Mora, Y. (2012). Formación permanente del docente de inglés: una experiencia exitosa en Costa Rica. Revista Iberoamericana sobre Calidad, Eficacia y Cambio en Educación, 10(4), 103-113. https://dialnet.unirioja .es/servlet/articulo?codigo $=4132513$

Calvete, M. y Laurentis, C. (2014). Una experiencia en la formación de docentes de inglés: Pedagogía Crítica y Estudios Poscoloniales. Revista de Educación, 5(7), 229- 242. https://bit.ly/2K39mw8

Cárdenas, M. L. , González, A. y Aldemar, J. (2010). El desarrollo profesional de los docentes de inglés en ejercicio: algunas consideraciones conceptuales para Colombia. Segunda época, (31), 49-68. https://dialnet.unirioja.es/se rvlet/articulo?codigo $=3790471$

Cedeño-Álava, M. A., Loor-Lara, D. L., Ponce-Martínez, R. I. y Loor-Domo, M. L. (2020). La enseñanza de la asignatura inglés desde una concepción problémica sustentada en el enfoque comunicativo. Polo del Conocimiento: Revista cientifico-profesional, 5(2), 250-272. https://dialnet.unirioja.es/servlet/articulo?codigo= 7435326

Chávez-Zambrano, M., Saltos-Vivas, M. y Saltos-Dueñas, C. (2017). La importancia del aprendizaje y conocimiento del idioma inglés en la enseñanza superior. Revista Cientifica Dominio de las Ciencias, 3(3), 759-771. https://d ialnet.unirioja.es/servlet/articulo?codigo $=6234740$

Córdoba, P., Rodríguez, X. y Hernández, T. (2015). The Design and Implementation of an In-Service EFL Teacher Training Model in the Costa Rican Public School System. Gist Education and Learning Research Journal, (10), 29-50. https://files.eric.ed.gov/fulltext/EJ1070670.pdf

Correa, O. (2019). Revaloración de enfoques tradicionales en la enseñanza de lenguas adicionales: Resistencias y oportunidades de docencia creativa. DOCERE, (21), 20-23. https://revistas.uaa.mx/index.php/docere/article/ view/2477

Cortina, G. F. (2013, 18-22 de noviembre). La enseñanza del idioma inglés en las instituciones de educación superior federalizadas del estado de México [Ponencia]. XII Congreso Nacional de Investigación Educativa, Guanajuato, Guanajuato, México. http://www.comie.org.mx/congreso/memoriaelectronica/v12/doc/0429.pdf

Cruz, H., Reyes, C. y Frías, M. C. (2016). ¿Cómo enseñas inglés? Enfoque comunicativo, una herramienta eficaz (pp. 120-131). En R. Velázquez, F.J. Linares y M.L. Quintero (Ed.), La Inclusión Educativa. UAEMEX.

Dávila, G. P., Damián, D. L., Chamorro, C. P. y Plua, K. A. (2020). Evaluación de las habilidades escritas en inglés y su desarrollo. Ciencia Digital, 4(1), 292-303. https://cienciadigital.org/revistacienciadigital2/index.php/Cienc iaDigital/article/view/1102 
Del Campo, M. (2012). La didáctica no parametral: hacia un nuevo paradigma en la formación de docentes de inglés. Porta Linguarium, 18, 133-148. https://dialnet.unirioja.es/servlet/articulo?codigo $=4593171$

Dulzaides, M. E. y Molina, A. M. (2004). Análisis documental y de información: dos componentes de un mismo proceso. Acimed: revista cubana de los profesionales de la información y la comunicación en salud, 12(2), 1-5. htt ps://bit.ly/3hT2NbY

Echeverri, P. A., Arías, N. y Gómez, I. C. (2014). La Pedagogía Crítica en la Formación de Docentes de Inglés: La Experiencia de un Grupo de Estudio. Íkala, Revista de Lenguaje y Cultura, 19(2), 167-181. https://revistas.ud ea.edu.co/index.php/ikala/article/view/17712

Espejo-Mohedano, A. R. y Palacios-Hidalgo, F. J. (2019). Webs y aprendizaje de lenguas: análisis de actividades reading y listening para el hablante no nativo de inglés: el caso de la BBC. EDMETIC, Revista de Educación Mediática y TIC, 8(1), 72-87. https://dialnet.unirioja.es/servlet/articulo?codigo $=6775505$

Estrada, M. E. y García, C. (2018). Alfabetización académica y crítica en la formación de docentes de inglés. En M. R. Espinosa y J. R. Díaz (Eds.). Propuestas docentes en el aprendizaje de idiomas y las ciencias. Congreso Internacional de Transformación Educativa Prospectiva y Emancipación Social: Aprendizaje Creador (pp. 45-57). Amapsi. htt ps://www.academia.edu/41575941

Farell, T. S. C. (2015). Second Language Teacher Education: A Reality Check. Palgrave. https://bit.ly/3ottTc9

Fierro, L. E., Martínez, L. y Román, R. D. (2016). Evaluación del aprendizaje del inglés desde dos perspectivas de formación. Debates en Evaluación y Curriculum, Congreso Internacional de Educación Evaluación, 2(2), 1106-1126. https://posgradoeducacionuatx.org/ano5no5.html

Galvañy, M. M. y Martín, Y. (2011). ¿Se puede desarrollar la competencia comunicativa utilizando un juego didáctico de mesa? Cuadernos de Educación y Desarrollo, 3(27). https://bit.ly/39dJwxY

García-Valcárcel, A., Basilotta, V. y Mulas, I. (2016). Fomentando la ciudadanía digital mediante un proyecto de aprendizaje colaborativo entre escuelas rurales y urbanas para aprender inglés. Profesorado, revista de currículum y formación del profesorado, 20(3), 549-581. https://www.redalyc.org/articulo.oa?id=56749100019

Garton, S., Copland, F. y Burns, A. (2011). Investigating Global Practices in Teaching English to Young Learners. Atson University.

Ghaffari, M., Kashkouli, S. M. y Sadighi, F. (2017).Montessori and Conventional Teaching Methods in Learning English as a Second/Foreign Language: An Overview. Journal of Applied Linguistics and Language Research, 4(5), 209-218. http://www.jallr.com/index.php/JALLR/article/view/657

Gómez-Parra, M. E. y Huertas-Abril, C. (2019). La importancia de la competencia digital para la superación de la brecha lingüística en el siglo XXI: Aproximación, factores y estrategias. EDMETIC, Revista de Educación Mediática y TIC, 8(1), 88-106. https://dialnet.unirioja.es/servlet/articulo?codigo $=6775508$

González, M. C. y Moyetta, M. V. (2014). Moodle como herramienta de apoyo en la enseñanza del inglés para turismo desde el enfoque comunicativo. Virtualidad, Educación y Ciencia, 5(9), 70-77 https://revistas.unc.edu.ar/inde x.php/vesc/issue/view/902

Guevara, R. (2016). El estado de arte en la investigación: eanálisis de los conocimientos acumulados o indagación por nuevos sentidos? Folios Segunda Época, 44,165-179. https://www.redalyc.org/articulo.oa?id=345945922011

Gutiérrez, M. y Landeros, I. A. (2010). La importancia del lenguaje en el contexto de la aldea global. Horizontes Educacionales, 15(1), 95-107. https://www.semanticscholar.org/paper/Importancia-del-lenguaje-en-el-context o-de-la-aldea-Ram\%C3\%ADrez-Falc\%C3\%B3n/3298809ed4cdc7b4596c18799005726dd7919d0d

Hinkel, E. (2011). What Research on Second Language Writing Tells Us and What it Doesn't. En E. Hinkel (Ed.), Research in Second Language Processes and Development (pp.523-538). Routledge. https://www.researchgate.net/publication/265408667_What_Research_on_Second_Language_ Writing_Tells_Us_and_What_it_Doesn $\% 27 \mathrm{t}$

Janssen, G., Nausa, R. y Rico, C. (2012). Shaping the ESP curriculum of an English for PhD students program: a colombian case study of questionnaire research. Colombian Applied Linguistics Journal, 14(2), 51-69. http://w ww.redalyc.org/articulo.oa?id=305726043006 
Jenkins, J., Cogo, A. y Dewey, M. (2011). Review of developments in research into English as a lingua franca. Language Teaching, 44(3), 281-315. https://bit.ly/2XlCFwH

Laurentis, C. y Porta, L. (2014). Voces de alumnos y docentes: narrativas como espacios contrapúblicos en la carrera del profesorado de Inglés. Praxis Educativa, 18(1), 32-41. http://www.redalyc.org/articulo.oa?id=153137899003

Lin, A. (2008). Cambios de paradigma en la enseñanza de inglés como lengua extranjera: el cambio crítico y más allá. Revista Educación y Pedagogia, 22(51), 11-23. https://dialnet.unirioja.es/servlet/articulo?codigo=3074193

López, R., Salas, J. y Quesada, M. (2017). Nueva propuesta curricular para la Universidad de Costa Rica: inglés con formación en gestión empresarial. Revista Congreso Universidad, 6(2), http://revista.congresouniversidad.cu/in dex.php/rcu/article/view/816

Luna-Hernández, A., Ortiz-Deliz, F. y Rey-Rivas, P. (2014). El enfoque comunicativo inmerso en el aprendizaje del idioma inglés en ciencia de la computación. Editorial Universitaria de la República de Cuba, (134), 548-558. ht tps://santiago.uo.edu.cu/index.php/stgo/article/view//230

Marcos, K. (1997). Why, how and when should my child learn a second language? Eric.

Marmolejo, M. y Mayora, C. (2020). El currículo del área de inglés de cuatro instituciones educativas oficiales de Cali: un estudio de caso múltiple. Revista Boletín Redipe, 99(22),108-131. https://revista.redipe.org/index.php/1/a rticle/view/914

Martínez, R. E., Flores, M. A. y Paz, K. R. (2018). Rúbricas en el proceso de evaluación de la expresión oral en inglés. Revista Atlante: Cuadernos de Educación y Desarrollo, 1-15. http://www.eumed.net/rev/atlante/2018/01/expr esion-oral-ingles.html

Martínez-Salas, M. M. (2019). El modelo pedagógico de clase invertida para mejorar el aprendizaje del idioma inglés. Investigación Valdizana, 13(4), 204-213. https://www.redalyc.org/journal/5860/586062183003/html/index. html

Mayora, C. A. y Gutiérrez, D. M. (2019). Análisis del currículo del área de inglés en escuelas oficiales de Cali. Lenguaje, 47(25), 670-685. http://www.scielo.org.co/scielo.php?pid=S0120-34792019000300685\&script=sci_abstract $\& \mathrm{t} \operatorname{lng}=\mathrm{es}$

Medina, M. (2011). El estado del Arte. Revista de Investigación Scientia Educere, 1, 1-13.

Mena, N. P., Abata, F. M. y Rosero, J. L.(2016). La andragogía y las estrategias metodológicas en el aprendizaje del idioma inglés en adultos.Didasc@alia:Didáctica y Educación, 7(6),101-114. https://dialnet.unirioja.es/servle $\mathrm{t} /$ articulo?codigo $=6672793$

Ming, A.L. (2012). La ubicación de la gramática (explícita) en el seno de los métodos y enfoques de enseñanza de las lenguas extranjeras: ‘al norte, en el centro o en las afueras? Onomázein, (25), 107-124. http://www.redalyc.org /articulo.oa?id=134524361005

Mogrovejo, A. B., Mamani, G. y Tipo, M. L. (2019). Juego y Simulación de Programas Concurso de Televisión como Técnica Didáctica para Mejorar el Aprendizaje del Vocabulario Inglés en Estudiantes de Habla Hispana. Información Tecnológica, 30(1), 225-236. https://www.scielo.cl/scielo.php?script=sci_arttext\&pid=S0718-07 642019000100225\&lng=en\&nrm=iso\&tlng=en

Mora, R. A. (2011). Tres retos para la investigación y formación de docentes en inglés: reflexividad sobre las creencias y prácticas en literacidad.Revista Educación, Comunicación y Tecnología, 5(10), 1-20.https://bit.ly/3otWAWr

Muñoz, A. (2010). Metodologías para la enseñanza de lenguas extranjeras. Hacia una perspectiva crítica. Revista Universidad EAFIT, 46(159), 71-85. https://publicaciones.eafit.edu.co/index.php/revista-universidad-eafit/ar ticle/view/1065

Naranjo, M. y Naranjo, X. (2017). Estrategias metodológicas para potenciar el aprendizaje de idioma inglés como lengua extranjera en la Unidad educativa Guayaquil-Ambato. Revista Publicando, 12(1), 164-173. https://revi stapublicando.org/revista/index.php/crv/article/view/648

Noroozisiam, E. y Soozandehfar, S. (2011). Teaching English through Critical Pedagogy: Problems and Attitudes. Theory and Practice in Language Studies, 1(9), 1240-1244. https://doi.org/10.4304/tpls.1.9.1240-1244

Ochoa-Vásquez, M. A. y Ramírez-Montoya, M. S. (2017). Técnicas de evaluación de comprensión lectora en inglés con formación mediada por tecnologías para mejorar el rendimiento estudiantil universitario. Revista electrónica 
calidad en la educación superior, 8(1), 225-250. https://revistas.uned.ac.cr/index.php/revistacalidad/article/vie $\mathrm{w} / 1787$

Olufunmilyo, J. (2014). The State of English Language Teaching and Learning in Nigeria and Namibia: Implications for National Development. International Journal of Innovate Research and Studies, 3(4), 219-249. https://bit. ly/35nqOTo

Oros, A., Ortega, D., Monzón, M. y Sarango, F. (2018). El aprendizaje activo para las clases de inglés como lengua extranjera. Observatorio de la Educación-UNAE, Cuaderno de Politica Educativa 3, 1-14. http://repositorio.un ae.edu.ec/handle/56000/450

Ortega-Auquilla, D. P. y Minchala-Buri, O. E. (2019). Explorando las Aulas de Clase de Inglés en Cañar: Currículo, instrucción y aprendizaje. CIENCIA UNEMI, 12(30), 57-73. https://www.redalyc.org/journal/5826/582661 249006/index.html

Ortiz-Navarrete, M. y Díaz-Larenas, C. (2017). Contribuciones teóricas sobre la metodología colaborativa para el tratamiento de los errores gramaticales de un texto académico en inglés en un contexto universitario. Revista Electrónica Educare, 21(2), 1-11. https://bit.ly/2LdOqmA

Ouyang, H. (2000). One-way ticket: a story of an innovative teacher in Mainland China. Antropology and Education Quarterly, 31(4), 397-425. https://www.jstor.org/stable/3196040

Paineán, O., Torres, T. y Aliaga, V. (2012). Aprendizaje basado en problemas: evaluación de una propuesta curricular para la formación inicial docente. Estudios Pedagógicos, 38(1), 168-180. http://dx.doi.org/10.4067/S0718-070 52012000100010

Patarroyo, E., Díaz, J. C. y Barreto de Quintero, S. (2004). ¿Es el currículo oculto un aspecto importante en los procesos educativos? Profile, 5(1), 162-165. https://dialnet.unirioja.es/servlet/articulo?codigo $=4858671$

Pinto, M. (1989). Introducción al análisis documental y sus niveles: análisis de contenido. Boletín de la ANABAD, 2, 323-342. https://dialnet.unirioja.es/servlet/articulo?codigo $=798857$

Quesada, M. J. (2015). Creación de videos educativos como estrategia didáctica para la formación de futuros docentes de inglés. Revista Actualidades Investigativas en Educación, 15(1), 1-19. https://www.scielo.sa.cr/pdf/aie/v15n 1/a06v15n1.pdf

Quezada-Narvaéz, C. (2011). La popularidad del inglés en el siglo XXI. Tlatemoani Revista Académica de Investigación, 5. https://dialnet.unirioja.es/servlet/articulo?codigo $=7313478$

Ramírez, J. L., Pamplón, E. N y Cota, S. (2012). Problemática de la enseñanza del inglés en las primarias públicas de México, una primer lectura cuantitativa. Revista Iberoamericana de Educación, 60(2), 1-12. https://dialnet.uni rioja.es/servlet/articulo?codigo $=5892599$

Recino, U., Quesada, A., Finalet, E. y González, M. F. (2014). Herramienta metodológica para desarrollar competencia comunicativa en idioma inglés en las universidades médicas cubanas. Edumecentro, 6(2), 214-230. http://scielo .sld.cu/scielo.php?script $=$ sci_arttext\&pid=S207728742014000200015

Richards, J. C. (2001). Curriculum Development in Language Teaching. Cambridge University Press.

Rivera, P. y Garrote, J. (2017). Uso formativo de instrumentos de evaluación en secundaria, lengua extranjera Inglés: redacciones. Infancia, Educación y Aprendizaje (IEYA), 3(2), 363-368. https://revistas.uv.cl/index.php/IEYA/ article/view/750

Rodríguez, R. y Gómez, M. (2017). Competencias digitales en el aprendizaje del inglés en bachillerato. Campus Virtuales, 6(2), 51-59. http://uajournals.com/ojs/index.php/campusvirtuales/article/view/185/199

Rojas, M. J. y Montes de Oca, B. I. (2018). Dinamizando la metodología b-learning para la enseñanza del inglés. Unidades didácticas mediadas por TIC. En: R. Roig-Vila (Ed.). El compromiso académico y social a través de la investigación e innovación educativas en la Enseñanza Superior. (pp. 425-437). Octaedro. http://rua.ua.es/dspa ce/handle/10045/84990

Rojas, M.J. (2018). La lingüística descriptiva y aplicada a la enseñanza de lenguas: insumos teóricos para una propuesta curricular en un programa de pregrado en bilingüismo con énfasis español-inglés. Voces y Silencios: Revista Latinoamericana de Educación, 9(1), 114-133. https://revistas.uniandes.edu.co/doi/pdf/10.18175/vys9.1.201 8.07 
Roldán, R. J. (2018). Programa "B-English", basado en clases semipresenciales, para el dominio de las habilidades comunicativas del idioma inglés. Rev. Cienc. Tecnol., 14(3), 99-106. https://revistas.unitru.edu.pe/index.php/ PGM/article/view/2110

Ruíz, U., Ampudia, M. P. y Caballero, M. (2020). La lengua inglesa y francesa en el currículum de la Licenciatura en Lenguas. Identidad Universitaria, 1(8), 13-15. https://revistaidentidad.uaemex.mx/article/view/14202

Sagástegui, E .E., Vilca, L. M. y González, A. C. (2018). Estrategias de enseñanza-aprendizaje para mejorar la producción oral en inglés de los estudiantes de la Escuela de Idiomas de la UCV-2017. UCV-Scienta, 10(01), 75-81. http://revistas.ucv.edu.pe/index.php/UCV-SCIENTIA/article/view/1770

Salmani-Nodoushan, M. A. (2006). Language Teaching: State of the Art. The Asian EFL Journal Quaterly, 8(1), 169-193. https://bit.ly/3s7j1Tv

Sarasa, M.C. (2017). A Narrative Inquiry Into Pre-Service English Teachers' Temportal Investments in Their Initial Education Curriculum. HOW Journal, 24(1), 27-43. https://www.howjournalcolombia.org/index.php/how/a rticle/view/337

Sarasa, M. C. (2018). Narrativas vitales en las socialidades del currículo universitario para la formación inicial del profesorado. Revista Brasileira de Pesquisa (Auto)Biográfica, 3(8), 441-461. https://bit.ly/2XIElX1

Sarmiento, M., Batista, T. y Jaramillo, Y. (2017, 26-28 de abril). Los cursos deposgrado de idioma inglés en función de las necesidades de la sociedad. [Ponencia]. Conferencia Científica Internacional, Octava Edición. Holguín, Cuba. h ttps://eventos.uho.edu.cu/index.php/ccm/cci8/schedConf

Smith, R. y McLelland, N. (2018). Histories of language learning and teaching in Europe. The Language Learning Journal, 46(1), 1-15. https://doi.org/10.1080/09571736.2017.1382051

Tejeda, I. y Borrero, V. V. (2019). El enfoque basado en tareas en los cursos de inglés del posgrado. Didasc@lia: Didáctica y Educación, 10(4), 85-96. https://dialnet.unirioja.es/servlet/articulo?codigo=7248599

Thomson, R. y Derwing, T. (2014). The Effectiveness of L2 Pronunciation Instruction: A Narrative Review. Applied Linguistics, 1-20. https://bit.ly/3s8TBVq

Tobón, S., Guzmán, C. E., Silvano, J. y Cardona, S. (2015). Sociedad del Conocimiento: Estudio documental desde una perspectiva humanista y compleja. Revista Paradigma, 36(2), 7-36. https://bit.ly/39cOBXo

Vargas, S. (2017). Promoviendo la pedagogía crítica en la enseñanza del inglés: experiencia de práctica pedagógica. Revista Huellas, 1(8), 46-54. https://revistas.udenar.edu.co/index.php/rhuellas/article/view/3452

Zambrano, J. A., Carrera, G.M., Williams, M. S., Venegas, G. S. y Bazurto, G. J. (2017). Blended learning como estrategia de enseñanza-aprendizaje del idioma inglés.Didasc@alia:Didáctica y Educación, 9(1), 55-70. https:/ $/$ dialnet.unirioja.es/servlet/articulo?codigo $=6595068$

Zambrano, G. E. y Mendoza, R. E. (2018). Influencia del método B-learning en la enseñanza-aprendizaje del inglés en la comunidad educativa de la Universidad Laica Eloy Alfaro de Manabí, extensión Chone. Universidad y Sociedad, 10(1), 255-262.http://scielo.sld.cu/scielo.php?script=sci_abstract\&pid=S2218-36202018000100255

Zepeda-Hernández, S., Abascal-Mena, R. y López-Ornelas, E. (2016). Integración de gamificación y aprendizaje activo en el aula. Ra Ximhai, 12(6), 315-325. https://www.redalyc.org/articulo.oa?id=46148194022

\section{INFORMACIÓN ADICIONAL}

Cómo citar: Arán-Sánchez, A., Arzola-Franco, D. M. y Ríos-Cepeda, V. L. (2022). Enfoques en el currículo, la formación docente y metodología en la enseñanza y aprendizaje del inglés: una revisión de la bibliografía y análisis de resultados. Revista Educación, 46(1). http://doi.org/10.15517/revedu.v46i1.45048 